\title{
NEW APPROACHES FOR VIRTUAL PRIVATE NETWORK DESIGN*
}

\author{
FRIEDRICH EISENBRAND ${ }^{\dagger}$, FABRIZIO GRANDONI ${ }^{\ddagger}$, GIANPAOLO ORIOLO $^{\S}$, AND \\ MARTIN SKUTELLA
}

\begin{abstract}
Virtual private network design is the following NP-hard problem. We are given a communication network represented as a weighted graph with thresholds on the nodes which represent the amount of flow that a node can send to and receive from the network. The task is to reserve capacities at minimum cost and to specify paths between every ordered pair of nodes such that all valid traffic-matrices can be routed along the corresponding paths. Recently, this network design problem has received considerable attention in the literature. It is motivated by the fact that the exact amount of flow which is exchanged between terminals is not known in advance and prediction is often elusive. The main contributions of this paper are as follows: (1) Using Hu's 2-commodity flow theorem, we provide a new and considerably stronger lower bound on the cost of an optimum solution. With this lower bound we reanalyze a simple routing scheme which has been described in the literature many times, and provide an improved upper bound on its approximation ratio. (2) We present a new randomized approximation algorithm. In contrast to earlier approaches from the literature, the resulting solution does not have tree structure. A combination of our new algorithm with the simple routing scheme yields an expected performance ratio of 3.79 for virtual private network design. This is a considerable improvement of the previously best known 5.55-approximation result [A. Gupta, A. Kumar, and T. Roughgarden, Simpler and better approximation algorithms for network design, in Proceedings of the ACM Symposium on Theory of Computing, ACM, New York, 2003, pp. 365-372]. (3) Our VPND algorithm uses a Steiner tree approximation algorithm as a subroutine. It is known that an optimum Steiner tree can be computed in polynomial time if the number of terminals is logarithmic. Replacing the approximate Steiner tree computation with an exact one whenever the number of terminals is sufficiently small, we finally reduce the approximation ratio to 3.55. To the best of our knowledge, this is the first time that a nontrivial result from exact (exponential) algorithms leads to an improved polynomial-time approximation algorithm.
\end{abstract}

Key words. approximation algorithms, randomized algorithms, network design

AMS subject classifications. $68 \mathrm{~W} 20,68 \mathrm{~W} 25$

DOI. $10.1137 / 060654827$

1. Introduction. Consider a communication network which is represented by an undirected graph $G=(V, E)$ with edge-weights $c: E \rightarrow \mathbb{R}_{+}$. Within this network there is a set of terminals $T \subseteq V$ which want to communicate with each other. However, the exact amount of traffic between pairs of terminals is not known in advance. Instead, each terminal $v \in T$ has associated input and output thresholds $b_{\text {in }}(v) \in \mathbb{Z}_{\geq 0}$ and $b_{\text {out }}(v) \in \mathbb{Z}_{\geq 0}$. A traffic-matrix $D \in \mathbb{Q}_{\geq 0}^{T T}$ is valid if it respects the lower and upper bounds on the incoming and outgoing traffic of the terminals, i.e., if

* Received by the editors March 22, 2006; accepted for publication (in revised form) February 2, 2007; published electronically June 29, 2007. Preliminary versions of these results appeared in the Proceedings of SODA'05 [7] and of ICALP'05 [8].

http://www.siam.org/journals/sicomp/37-3/65482.html

$\dagger$ Fachbereich Informatik, Universität Dortmund, 44221 Dortmund, Germany (friedrich. eisenbrand@cs.uni-dortmund.de).

¥Dipartimento di Informatica, Università di Roma "La Sapienza," Via Salaria 113, 00198 Roma, Italy (grandoni@di.uniroma1.it).

§Dipartimento di Ingegneria dell'Impresa, Università di Roma "Tor Vergata," Via del Politecnico 1, 00165, Roma, Italy (oriolo@disp.uniroma2.it).

『Fachbereich Mathematik, Universität Dortmund, 44221 Dortmund, Germany (martin.skutella@ uni-dortmund.de). 
the following holds for each terminal $i \in T$ :

$$
\sum_{j \in T, j \neq i} D(i, j) \leq b_{\text {out }}(i) \quad \text { and } \quad \sum_{j \in T, j \neq i} D(j, i) \leq b_{\text {in }}(i) .
$$

The (asymmetric) virtual private network design (VPND) problem defined by $G$, $c$, and $T$ consists of finding capacities $u(e), e \in E$ and paths $P_{i j}$ for each ordered pair $(i, j) \in T T$ such that the following conditions hold:

(i) All valid traffic-matrices can be routed without exceeding the installed capacities where all traffic from terminal $i$ to terminal $j$ is routed along path $P_{i j}$.

(ii) The total cost of the reservation $\sum_{e \in E} u(e) c(e)$ is minimal.

A reservation of capacities $u: E \rightarrow \mathbb{R}_{+}$is a tree reservation if the subgraph of $G$ induced by the edges $e \in E$ with $u(e)>0$ is a tree. A general reservation is sometimes referred to as a graph reservation.

The virtual private network design problem is NP-hard by the following reduction from the Steiner tree problem [11]. Given an instance of the Steiner tree problem, pick a terminal which has to be connected with the other terminals in a Steiner tree. This terminal has thresholds $b_{i n}(v)=0$ and $b_{\text {out }}(v)=1$. All other terminals $u$ of the Steiner tree instance have $b_{\text {in }}(u)=1$ and $b_{\text {out }}(u)=0$. A minimum cost Steiner tree also yields an optimum reservation for this VPND instance.

The virtual private network design problem was independently defined by Fingerhut, Suri, and Turner [10] and by Duffield et al. [6] and has since then been studied by various authors in several variations which we next discuss. In the following list, the last one $($ Asym $G)$ is the one which we refer to as VPND.

$($ SymT) Symmetric thresholds, tree reservation: In this variant, each terminal $i \in T$ has only one threshold $b(i)$, which is an upper bound on the cumulative amount of traffic that terminal $i$ can send or receive. The task is to find an optimal tree reservation which supports all valid traffic-matrices. Gupta et al. [11] show that $(S y m T)$ is polynomially solvable.

$($ Sym $G)$ Symmetric thresholds, graph reservation: This variant is defined in the same way as $(S y m T)$, except that the capacity reservation can be arbitrary and not necessarily a tree. Gupta et al. [11] present a 2-approximation for $(S y m G)$. It is not known whether $\operatorname{Sym} G$ is NP-hard.

(BalT) Balanced thresholds, tree reservation: The thresholds are balanced, which means that $\sum_{v \in T} b_{i n}(v)=\sum_{v \in T} b_{\text {out }}(v)$. The reservation has to be a tree. Italiano, Leonardi, and Oriolo [15] show that this variant can be solved in polynomial time.

(BalG) Balanced thresholds, graph reservation: The same as (BalT), except that an arbitrary graph reservation is allowed.

(AsymT) Asymmetric thresholds, tree reservation: This problem is NP-hard [11]. Constant approximation algorithms are presented in [11, 12]. Interestingly, while the algorithm in [11] is deterministic, the algorithm in [12] is randomized and seems difficult to derandomize.

$($ Asym $G)$ Asymmetric thresholds, graph reservation: This is the VPND problem defined above. We have seen that this problem is NP-hard. The randomized 5.55 -approximation result presented in [12] in fact compares the computed tree solution to an optimal graph reservation.

Simplifying assumptions and a lower bound. Following [12], we make some simplifying assumptions without loss of generality. By duplicating nodes, we can assume that each terminal is either a sender $s$, with $b_{\text {out }}(s)=1$ and $b_{\text {in }}(s)=0$, or a 
receiver $r$, with $b_{\text {out }}(r)=0$ and $b_{\text {in }}(r)=1$. This simplifying assumption is feasible as long as we make sure that the selected paths in our solution between copies of a terminal $v$ and copies of a terminal $u$ are all equal. The algorithms presented in this paper can easily be adapted to run in polynomial time even when the thresholds are not polynomially bounded and to satisfy the consistence property described above. Let $\mathcal{S}$ and $\mathcal{R}$ be the set of senders and the set of receivers, respectively. By $S=|\mathcal{S}|$ and $R=|\mathcal{R}|$ we denote the corresponding cardinalities. For symmetry reasons, we always assume $R \geq S$.

We can now interpret VPND as follows. Let $B=\left(\mathcal{S} \cup \mathcal{R}, E^{B}\right)$ be the complete bipartite graph with nodes partitioned into senders and receivers. We have to reserve capacities on the edges of $G$ and we have to specify a set of paths $\mathcal{P}$ in graph $G$ containing one path $P_{s r}$ for each edge $s r \in E^{B}$ such that each bipartite matching of $B$ can be routed along these paths. In other words, for each edge $e \in E$, the reservation $u(e)$ has to satisfy the following condition:

$$
\mid\left\{P_{r s} \in \mathcal{P} \mid e \in P_{r s} \text { and } r s \in M\right\} \mid \leq u(e) \text { for each matching } M \text { in } B .
$$

Notice that for a fixed set of paths $\mathcal{P}$, an optimal reservation of capacity is the componentwise minimal $u$ satisfying (1). (In particular, given $\mathcal{P}$, the integral capacity $u(e)$ of edge $e$ can be obtained by a maximum bipartite matching computation.) Thus, a solution to VPND can be encoded by specifying only a set of paths $\mathcal{P}$ in $G$.

The cost of a bipartite matching between senders and receivers in the metric closure of $G$ is obviously a lower bound on $O P T$, the value of an optimum solution to the VPND instance. We denote the shortest path distance between nodes $u$ and $v$ of $G$ by $\ell(u, v)$. Thus, if edges $(r, s)$ in $B$ are assigned weights $\ell(r, s)$, then the cost of any matching in $B$ is a lower bound on $O P T$. This lower bound is used in the analysis of all previous constant-factor approximation algorithms for VPND.

Lemma 1 (see [12]). Let $B=\left(\mathcal{S}+\mathcal{R}, E^{B}\right)$ be the complete bipartite graph on the senders and receivers with edge-weights $\ell: E^{B} \rightarrow \mathbb{R}_{+}$given by the shortest path distances in the graph $G$. Then, the weight of any matching in $B$ is a lower bound on $O P T$.

Contribution of this paper. The design of good approximation results usually requires two main ingredients - cleverly constructed algorithms and thoroughly chosen lower bounds on the optimum such that the quality of the computed solutions can be assessed in terms of the lower bounds. We considerably advance the state of the art of approximating VPND by making contributions to both ingredients.

In section 2 we present a new lower bound which strengthens the one stated in Lemma 1. We prove that the weight of any matching (not necessarily bipartite) on the union of the senders and at most $S$ receivers is at most $O P T$. The edge-weights in the matching are again the shortest path distances in $G$. This new lower bound relies on an interesting interrelation between a special case of VPND and 2-commodity flows. Its proof is based on an application of Hu's 2-commodity flow theorem [13].

In section 3 we employ the new lower bound in order to show that the following simple algorithm achieves performance ratio $1+R / S$ : Find a vertex $v \in V$ which minimizes the sum of the shortest path distances between $v$ and the union of senders and receivers; cumulatively install a capacity of one on each such shortest path. One interesting consequence of this result is that $(B a l G)$, VPND with balanced thresholds and graph reservation, has a 2-approximation. Thus our result improves upon the 3approximation of Italiano, Leonardi, and Oriolo [15] for this problem and generalizes the 2-approximation for $(\operatorname{Sym} G)$ by Gupta et al. [11]. 
In section 4 we present a new randomized algorithm for VPND. The algorithm chooses a random subset of receivers and connects each sender via its own Steiner tree to this subset. The remaining receivers are then connected to the randomly chosen subset of receivers by shortest paths. Due to the Steiner trees for each individual sender, the resulting solution has in general no tree structure. In contrast to our new approach, the previous algorithm by Gupta, Kumar, and Roughgarden [12] constructs only one random "high-bandwidth core" which is a Steiner tree with high capacity. All previous approximation algorithms for VPND produce tree solutions.

In section 4 we show also that our new algorithm in combination with the simple algorithm from above yields a 3.79 randomized approximation algorithm. The previously best known algorithm [12] achieves performance ratio 5.55.

Our VPND algorithm uses a Steiner tree approximation algorithm as a subroutine. Dreyfus and Wagner [5] showed how to compute optimum Steiner trees in polynomial time when the number of terminals is logarithmic. In section 5, by replacing the approximate Steiner tree computation with an exact one whenever the number of terminals is sufficiently small, we eventually obtain a 3.55 -approximation algorithm. To the best of our knowledge, this is the first time a result from exact (exponential) algorithms leads to an improved polynomial-time approximation algorithm, which might be of independent interest.

Related work. We have seen that the Steiner tree problem is a special case of VPND. The current best approximation ratio for the Steiner tree problem is $\rho_{s t}<1.55$ [18]. A related problem is buy-at-bulk network design (see, e.g., [1, 2]). Here, there is a fixed demand $d_{i, j}$ between each pair of nodes in the graph, specifying the amount of flow which has to be sent from $i$ to $j$. The costs of the capacities however is a concave function on the amount purchased, which reflects "economies of scale." Gupta, Kumar, and Roughgarden [12] consider the single source buy-at-bulk network design problem and present a simple constant-factor approximation algorithm.

Another important issue in this context is to cope with arc failures in the network $[3,4]$. Italiano, Rastogi, and Yener [16] consider the problem of restoring the network, when at most one arc in a tree solution to VPND might fail and provide a constantfactor approximation algorithm.

It is conjectured that $(\operatorname{Sym} G)$ can be solved in polynomial time. It is in fact conjectured that an optimal solution to $(S y m T)$ is also an optimal solution to $(S y m G)$. Hurkens, Keijsper, and Stougie [14] show that this conjecture holds for rings. The authors also describe an integer programming formulation for VPND, which proves that a fractional version can be solved in polynomial time. This fractional version allows us to specify several paths for each sender-receiver pair and requires the fraction for each of these paths, which describes how the commodity has to be split.

2. A new lower bound via Hu's 2-commodity flow theorem. This section is devoted to proving a new lower bound on the cost of an optimal solution to VPND. Generalizing Lemma 1, we prove that the cost of an arbitrary (not necessarily bipartite) matching between terminals in $\mathcal{S} \cup \mathcal{R}^{\prime}$ is at most $O P T$ for any subset of receivers $\mathcal{R}^{\prime} \subseteq \mathcal{R}$ of cardinality $\left|\mathcal{R}^{\prime}\right|=S$. The proof of this result is based on Hu's classical 2-commodity flow theorem [13].

THEOREM 1 (Hu's 2-commodity flow theorem). Let $G=(V, E)$ be a graph and let $s_{1}, r_{1}, s_{2}, r_{2}$ be pairs of vertices of $G$; let $u: E \rightarrow \mathbb{R}_{+}$be a capacity function on the edges and let $d_{1}, d_{2} \in \mathbb{R}_{+}$. There exists a (fractional) 2-commodity flow of value $d_{1}, d_{2}$ if and only if the cut condition is satisfied, i.e., if and only if $u(\delta(U)) \geq$ 


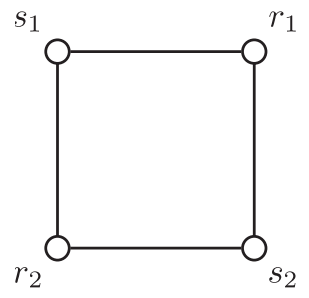

FIG. 1. An undirected graph with unit capacities on the edges. While there exist integral 2commodity flows satisfying unit demands for terminal pairs $\left\{s_{1}, r_{1}\right\},\left\{s_{2}, r_{2}\right\}$ and for terminal pairs $\left\{s_{1}, r_{2}\right\},\left\{s_{2}, r_{1}\right\}$, there is only a half-integral 2-commodity flow for terminal pairs $\left\{s_{1}, s_{2}\right\},\left\{r_{1}, r_{2}\right\}$.

$d_{1} \chi_{1}(U)+d_{2} \chi_{2}(U)$ for each $U \subseteq V$. Here $\delta(U)$ denotes the cut induced by $U$ and, for $i \in\{1,2\}, \chi_{i}(U)=1$ if the cut $\delta(U)$ separates $s_{i}$ from $r_{i}$, and $\chi_{i}(U)=0$ otherwise.

$\mathrm{Hu}$ 's theorem immediately implies the following corollary.

COROLlary 1 . Let $G=(V, E)$ be an undirected graph with edge capacity function $u: E \rightarrow \mathbb{R}_{+}$and $s_{1}, s_{2}, r_{1}, r_{2} \in V$. In the following, all demand values are equal to 1 . If there exists a feasible 2-commodity flow for terminal pairs $\left\{s_{1}, r_{1}\right\},\left\{s_{2}, r_{2}\right\}$ and for terminal pairs $\left\{s_{1}, r_{2}\right\},\left\{s_{2}, r_{1}\right\}$, then there also exists a feasible 2-commodity flow for terminal pairs $\left\{s_{1}, s_{2}\right\},\left\{r_{1}, r_{2}\right\}$.

Proof. Hu's 2-commodity flow theorem states that there exists a feasible 2commodity flow if and only if the "cut condition" is satisfied. In the case of unit demands, the cut condition says that, for all $U \subseteq V$, the capacity $u(\delta(U))$ of the cut induced by $U$ must be at least the number of terminal pairs which are separated by the cut.

It thus remains to show that the cut condition holds for terminal pairs $\left\{s_{1}, s_{2}\right\}$, $\left\{r_{1}, r_{2}\right\}$ if it holds for $\left\{s_{1}, r_{1}\right\},\left\{s_{2}, r_{2}\right\}$ and for $\left\{s_{1}, r_{2}\right\},\left\{s_{2}, r_{1}\right\}$. Consider an arbitrary $U \subseteq V$. If the corresponding cut separates neither $\left\{s_{1}, s_{2}\right\}$ nor $\left\{r_{1}, r_{2}\right\}$, nothing needs to be shown. If $\delta(U)$ separates one terminal pair, say, $\left\{s_{1}, s_{2}\right\}$, then it separates either $\left\{s_{1}, r_{1}\right\}$ or $\left\{s_{2}, r_{1}\right\}$ since $s_{1}$ and $s_{2}$ lie on different sides of the cut. In particular, the capacity of the cut is at least 1 . Finally, if $\delta(U)$ separates both terminal pairs $\left\{s_{1}, s_{2}\right\},\left\{r_{1}, r_{2}\right\}$, then it must separate either $\left\{s_{1}, r_{1}\right\}$ and $\left\{s_{2}, r_{2}\right\}$ or $\left\{s_{1}, r_{2}\right\}$ and $\left\{s_{2}, r_{1}\right\}$. In both cases the capacity of the cut is at least 2 .

We remark that Corollary 1 is no longer true if we replace "2-commodity flow" by "integral 2-commodity flow." A counterexample is given in Figure 1. Even, Itai, and Shamir show that finding an integer 2-commodity flow is NP-hard [9]. On the other hand, Hu's result states that there always exists a half-integral flow in this case. For a more detailed account of results we refer the reader to Schrijver's book [19, Chapter 71].

The next lemma shows that a partition of the senders and receivers into $k$-subsets each and the "addition" of the optimal solutions of the $k$ subproblems induced by the pairs of subsets provide a lower bound on the optimal solution.

Lemma 2. Suppose that $\mathcal{S}_{1}, \mathcal{S}_{2}, \ldots, \mathcal{S}_{k}$ is a partition of $\mathcal{S}$ and that $\mathcal{R}_{1}, \mathcal{R}_{2}, \ldots, \mathcal{R}_{k}$ is a partition of $\mathcal{R}$. Let $I_{i}$ be the VPND instance on graph $G$ with senders $\mathcal{S}_{i}$ and receivers $\mathcal{R}_{i}$, and let $O P T_{i}$ be the value of an optimal solution to instance $I_{i}$. Then the following holds:

$$
\sum_{i=1}^{k} O P T_{i} \leq O P T
$$

Proof. Let $\mathcal{P}$ be an optimal set of paths for the original VPND instance with 
resulting capacity reservation $u: E \rightarrow \mathbb{R}_{+}$. The subset $\mathcal{P}_{i} \subseteq \mathcal{P}$ of paths with endpoints in $\mathcal{S}_{i} \cup \mathcal{R}_{i}$ defines a solution to subinstances $I_{i}$ with capacity reservation $u_{i}: E \rightarrow \mathbb{R}_{+}$. It suffices to show that $u(e) \geq \sum_{i=1}^{k} u_{i}(e)$ for each edge $e \in E$.

It follows from (1) that for each $i=1,2, \ldots, k$

$$
u_{i}(e)=\max _{M_{i}} \mid\left\{P_{r s} \in \mathcal{P}_{i} \mid e \in P_{r s} \text { and } r s \in M_{i}\right\} \mid,
$$

where $M_{i}$ ranges over all bipartite matchings between senders $\mathcal{S}_{i}$ and receivers $\mathcal{R}_{i}$. We denote the matching for which the maximum is attained by $\tilde{M}_{i}$. Then, the disjoint union $\tilde{M}:=\bigcup_{i=1}^{k} \tilde{M}_{i}$ is a bipartite matching between senders $\mathcal{S}$ and receivers $\mathcal{R}$. It thus follows from (1) that

$$
\begin{aligned}
u(e) & \geq \mid\left\{P_{r s} \in \mathcal{P} \mid e \in P_{r s} \text { and } r s \in \tilde{M}\right\} \mid \\
& =\sum_{I=1}^{k} \mid\left\{P_{r s} \in \mathcal{P}_{i} \mid e \in P_{r s} \text { and } r s \in \tilde{M}_{i}\right\} \mid=\sum_{i=1}^{k} u_{i}(e)
\end{aligned}
$$

for each edge $e \in E$. This concludes the proof.

We are now ready to prove the following theorem which gives a new lower bound on the cost of an optimal VPND solution.

TheOREM 2. Consider an arbitrary subset $\mathcal{R}^{\prime} \subseteq \mathcal{R}$ of $S$ receivers. Let $M$ be a matching in the complete graph on $\mathcal{S} \cup \mathcal{R}^{\prime}$. Then

$$
\sum_{v w \in M} \ell(v, w) \leq O P T
$$

Proof. Let $\mathcal{S}=\left\{s_{1}, s_{2}, \ldots, s_{S}\right\}$ and $\mathcal{R}^{\prime}=\left\{r_{1}, r_{2}, \ldots, r_{S}\right\}$. It suffices to prove the claim for perfect matchings. Suppose that the matching consists of the edges

$$
\begin{gathered}
s_{1} s_{2}, s_{3} s_{4}, \ldots, s_{2 k-1} s_{2 k} \text { and } r_{1} r_{2}, r_{3} r_{4}, \ldots, r_{2 k-1} r_{2 k}, \\
\text { and } s_{2 k+1} r_{2 k+1}, s_{2 k+2} r_{2 k+2}, \ldots, s_{S} r_{S} .
\end{gathered}
$$

Consider the following partition of $\mathcal{S}$ and $\mathcal{R}^{\prime}$ into $S-k$ subsets $\mathcal{S}_{i}$ and $\mathcal{R}_{i}^{\prime}$ :

$$
\begin{array}{lr}
\mathcal{S}_{i}=\left\{s_{2 i-1}, s_{2 i}\right\}, \mathcal{R}_{i}^{\prime}=\left\{r_{2 i-1}, r_{2 i}\right\}, & 1 \leq i \leq k, \\
\mathcal{S}_{i}=\left\{s_{i}\right\}, \mathcal{R}_{i}^{\prime}=\left\{r_{i}\right\}, & 2 k+1 \leq i \leq S .
\end{array}
$$

By Lemma 2, the sum of the optimum solutions $O P T_{i}$ of the VPND subinstances $I_{i}$ with senders $\mathcal{S}_{i}$ and receivers $\mathcal{R}_{i}^{\prime}$ is a lower bound on $O P T$. Thus we need only to prove that $\ell\left(s_{2 i-1}, s_{2 i}\right)+\ell\left(r_{2 i-1}, r_{2 i}\right) \leq O P T_{i}$ for each $1 \leq i \leq k$.

An optimum solution to the subproblem $I_{i}, 1 \leq i \leq k$, is a reservation of capacities that supports 2-commodity flows with unit demands for the terminal pairs $\left\{s_{2 i-1}, r_{2 i-1}\right\},\left\{s_{2 i}, r_{2 i}\right\}$ and for the terminal pairs $\left\{s_{2 i-1}, r_{2 i}\right\},\left\{s_{2 i}, r_{2 i-1}\right\}$. By Corollary 1, it must also support a 2-commodity flow for the terminal pairs $\left\{s_{2 i-1}, s_{2} i\right\}$, $\left\{r_{2 i-1}, r_{2 i}\right\}$. Therefore, the cost of this solution is at least $\ell\left(s_{2 i-1}, s_{2 i}\right)+\ell\left(r_{2 i-1}, r_{2 i}\right)$. This concludes the proof. 
3. The quality of a simple routing scheme. Consider the following simple VPND algorithm.

Algorithm 1 (simple routing scheme).

(1) Compute a vertex $v \in V$ such that $\sum_{s \in \mathcal{S}} \ell(s, v)+\sum_{r \in \mathcal{R}} \ell(r, v)$ is minimal.

(2) Add one unit of capacity along the shortest path between each $u \in \mathcal{R} \cup \mathcal{S}$ and $v$.

Algorithm 1 selects the vertex $v \in V$ which minimizes the sum of the distances from $v$ to the union of the senders and receivers, and reserves one unit of capacity along each of the shortest paths. Note that the effects of installing capacities along the shortest paths is cumulative. In other words, if $k$ shortest paths share the same edge, the algorithm adds $k$ units of capacity to that edge. Moreover, we assume the shortest paths are computed with a consistent tie-breaking rule. This way, the edges with nonzero capacity form a tree.

Algorithm 1 produces an optimal tree reservation in the symmetric case $(S y m T)$ [11] and in the balanced case (BalT) [15]. In the symmetric case, Gupta et al. [11] showed that the tree produced by the algorithm is a 2-approximate solution to the optimum graph reservation. Italiano, Leonardi, and Oriolo [15] show that, in the balanced case, the produced tree is a 3-approximate solution to the optimum graph reservation.

In this section, we apply our new lower-bound result to show that the algorithm produces a tree solution which is within $1+R / S$ from the optimum graph reservation. Thus $(B a l G)$ can also be approximated within a factor of two. We first need the following simple lower bound on $O P T$. Let $\ell\left(E^{\prime}\right):=\sum_{u v \in E^{\prime}} \ell(u, v)$ for any subset of edges $E^{\prime}$.

LEMma 3. The sum of the distances between each sender-receiver pair is at most $R$ times the optimum:

$$
\sum_{s \in \mathcal{S}} \sum_{r \in \mathcal{R}} \ell(s, r) \leq R \cdot O P T
$$

Proof. Let $B=\left(\mathcal{S}+\mathcal{R}, E^{B}\right)$ be the complete bipartite graph on the senders and receivers with edge-weights $\ell: E^{B} \rightarrow \mathbb{R}_{+}$given by the shortest path distances in the graph $G$. The edges of $B$ can be partitioned into a set $\mathcal{M}$ of $R$ matchings. By Lemma 1 , the cost $\ell(M)$ of each $M \in \mathcal{M}$ is at most $O P T$. Hence

$$
\sum_{s \in \mathcal{S}} \sum_{r \in \mathcal{R}} \ell(s, r)=\sum_{M \in \mathcal{M}} \ell(M) \leq R \cdot O P T .
$$

We are now ready to bound the approximation ratio provided by Algorithm 1 .

THEOREM 3. Algorithm 1 is a $(1+R / S)$-approximation algorithm for VPND.

Proof. Let $G^{m}=\left(\mathcal{R} \cup \mathcal{S}, E^{m}\right)$ be the metric closure of $\mathcal{R} \cup \mathcal{S}$, i.e., the complete graph on $\mathcal{R} \cup \mathcal{S}$ with edge-weight $\ell(u, v)$ given by the shortest path distance between $u$ and $v$ in $G$. We show that there exists a node $u \in \mathcal{R} \cup \mathcal{S}$ such that the cost of the complete star centered at $u$ satisfies

$$
\sum_{v \in \mathcal{R} \cup \mathcal{S}} \ell(u, v) \leq(1+R / S) O P T .
$$

If $R=S$, the edges of $E^{m}$ can be partitioned into $2 S-1$ perfect matchings. By Theorem 2, the weight of each matching is a lower bound on $O P T$. Since each edge 
is contained in exactly two stars of $G^{m}$,

$$
\sum_{v \in \mathcal{R} \cup \mathcal{S}} \sum_{u \in \mathcal{R} \cup \mathcal{S}} \ell(v, u)=2 \ell\left(E^{m}\right) \leq 2(2 S-1) O P T .
$$

Thus there must exist one star, whose weight is at most $2(2 S-1) O P T /(2 S)<2 O P T$.

Suppose for the remainder of the proof that $R>S$. In the following we denote by $\mathcal{M}_{S}$ and $\mathcal{M}_{\mathcal{R}}$ the set of matchings of cardinality at most $\lfloor S / 2\rfloor$ involving only senders and only receivers, respectively. Theorem 2 implies the inequality

$$
\ell\left(M_{\mathcal{S}}\right)+\ell\left(M_{\mathcal{R}}\right) \leq O P T \quad \text { for each } M_{\mathcal{S}} \in \mathcal{M}_{\mathcal{S}}, M_{\mathcal{R}} \in \mathcal{M}_{\mathcal{R}}
$$

In consideration of (3), we distinguish two cases.

(A) $\ell\left(M_{\mathcal{S}}\right) \leq O P T / 2$ for each $M_{\mathcal{S}} \in \mathcal{M}_{\mathcal{S}}$. Consider the subgraph $G_{\mathcal{S}}^{m}$ of $G^{m}$ which is induced by the senders. The edges $E_{\mathcal{S}}^{m}$ of $G_{\mathcal{S}}^{m}$ can be partitioned into $S$ matchings. Therefore

$$
\sum_{s^{\prime} \in \mathcal{S}} \sum_{s \in \mathcal{S}} \ell\left(s^{\prime}, s\right)=2 \ell\left(E_{\mathcal{S}}^{m}\right) \leq 2 S \frac{O P T}{2}=S O P T .
$$

Combining (4) and (2), one obtains

$$
\sum_{s^{\prime} \in \mathcal{S}}\left(\sum_{s \in \mathcal{S}} \ell\left(s^{\prime}, s\right)+\sum_{r \in \mathcal{R}} \ell\left(s^{\prime}, r\right)\right) \leq(S+R) O P T .
$$

Therefore there is a sender $s^{*}$ such that

$$
\sum_{v \in \mathcal{S} \cup \mathcal{R}} \ell\left(s^{*}, v\right) \leq(1+R / S) O P T .
$$

(B) $\ell\left(M_{\mathcal{S}}\right)>O P T / 2$ for some maximum weight matching $M_{\mathcal{S}} \in \mathcal{M}_{\mathcal{S}}$. Let $G_{\mathcal{R}}^{m}$ denote the subgraph of $G^{m}$ which is induced by the receivers. We will show below that, for any maximal matching $\tilde{M}$ in $G_{\mathcal{R}}^{m}$,

$$
\ell(\tilde{M}) \leq(R / S) O P T / 2 .
$$

Since the edges of $G_{\mathcal{R}}^{m}$ can be partitioned into at most $R$ maximal matchings, we can then argue in a similar manner as in case (A) that

$$
\sum_{r^{\prime} \in \mathcal{R}} \sum_{r \in \mathcal{R}} \ell\left(r, r^{\prime}\right) \leq 2 R \frac{R}{S} \frac{O P T}{2}=\left(R^{2} / S\right) O P T .
$$

Together with (2) this implies

$$
\sum_{r^{\prime} \in \mathcal{R}}\left(\sum_{s \in \mathcal{S}} \ell\left(s, r^{\prime}\right)+\sum_{r \in \mathcal{R}} \ell\left(r, r^{\prime}\right)\right) \leq\left(R+R^{2} / S\right) O P T,
$$

from which we conclude that there is a receiver $r^{*}$ satisfying

$$
\sum_{v \in \mathcal{S} \cup \mathcal{R}} \ell\left(v, r^{*}\right) \leq(1+R / S) O P T .
$$


It remains to prove (5). We distinguish between the two subcases in which $S$ is even and $S$ is odd.

(B.1) $S$ is even. Theorem 2 , together with the assumption $\ell\left(M_{S}\right)>O P T / 2$, implies $\ell\left(M_{\mathcal{R}}\right) \leq O P T / 2$ for each matching $M_{\mathcal{R}} \in \mathcal{M}_{\mathcal{R}}$. Note that $|\tilde{M}|=\lfloor R / 2\rfloor \geq S / 2$. Consider the $S / 2$ most expensive edges $M^{\prime}$ of $\tilde{M}$. Since $M^{\prime} \in \mathcal{M}_{\mathcal{R}}, \ell\left(M^{\prime}\right) \leq O P T / 2$. Hence the average cost of one edge of $\tilde{M}$ is upper bounded by $(O P T / 2) /(S / 2)=$ $O P T / S$. Since $\tilde{M}$ has at most $R / 2$ edges, we get

$$
\ell(\tilde{M}) \leq|\tilde{M}| \frac{O P T}{S} \leq \frac{R}{2} \frac{O P T}{S}=(R / S) O P T / 2 .
$$

(B.2) $S$ is odd. There is a sender $s^{*}$ which is missed by the maximum cost matching $M_{\mathcal{S}}$ of $G_{\mathcal{S}}^{m}$. For each matching $M_{\mathcal{R}} \in \mathcal{M}_{\mathcal{R}}$ and $r_{1}^{*} \in \mathcal{R}$ which is not matched by $M_{\mathcal{R}}$, Theorem 2 yields

$$
\ell\left(M_{\mathcal{S}}\right)+\ell\left(M_{\mathcal{R}}\right)+\ell\left(s^{*}, r_{1}^{*}\right) \leq O P T,
$$

and hence

$$
\ell\left(M_{\mathcal{R}}\right)+\ell\left(s^{*}, r_{1}^{*}\right) \leq O P T / 2 .
$$

Consider any other receiver $r_{2}^{*}$ which is not matched by $M_{\mathcal{R}}$. This receiver must exist since $R>S$. By the triangle inequality one has $\ell\left(r_{1}^{*}, r_{2}^{*}\right) \leq \ell\left(s^{*}, r_{1}^{*}\right)+\ell\left(s^{*}, r_{2}^{*}\right)$. As a result we get

$$
\ell\left(M_{\mathcal{R}}\right)+1 / 2 \ell\left(r_{1}^{*}, r_{2}^{*}\right) \leq O P T / 2
$$

for each matching $M_{\mathcal{R}} \in \mathcal{M}_{\mathcal{R}}$ and receivers $r_{1}^{*}, r_{2}^{*}$ which are missed by $M_{\mathcal{R}}$.

Now consider the $(S-1) / 2$ most expensive edges $M^{\prime}$ of $\tilde{M}$, and let $e^{\prime}$ be the next most expensive edge of $\tilde{M}$. Since $M^{\prime} \in \mathcal{M}_{\mathcal{R}}$, by (6),

$$
\ell\left(M^{\prime}\right)+1 / 2 \ell\left(e^{\prime}\right) \leq O P T / 2 .
$$

It follows that half the average cost of one edge of $\tilde{M}$ is upper bounded by $(O P T / 2) /(S-$ $1+1)=O P T /(2 S)$, from which we conclude

$$
\ell(\tilde{M}) \leq 2|\tilde{M}| \frac{O P T}{2 S} \leq 2 \frac{R}{2} \frac{O P T}{2 S}=(R / S) O P T / 2 .
$$

4. A new algorithm for VPND. In section 3 we described an algorithm which guarantees a good approximation ratio for $R$ close to $S$. Here we present a better approximation algorithm in case $R$ is sufficiently larger than $S$.

The algorithm by Gupta et al. [12] constructs one random "high-bandwidth core," i.e., a small Steiner tree with high capacity, where the terminals are a random sender and a random subset of receivers. Such a Steiner tree collects and distributes the demands from outside, and routes them along its high capacity paths. Our algorithm is also based on Steiner tree computations, but we proceed by connecting each sender to a previously sampled subset of receivers via $S$ distinct Steiner trees of low capacity, and by connecting the other receivers along their shortest paths to the sampled subset (see Figure 2). More precisely, our algorithm works as follows.

Algorithm 2.

(1) Partition $\mathcal{R}$ into $S$ subsets uniformly at random. Select one subset $\mathcal{R}^{\prime}$ uniformly at random, among the nonempty subsets in the partition. 

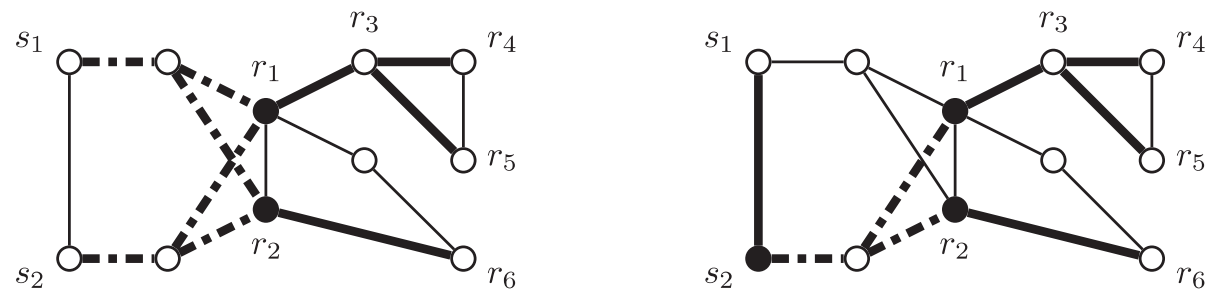

FIG. 2. Intuitive comparison between Algorithm 2 (on the left) and the algorithm in [12]. Black nodes form the randomly sampled subsets. Positive capacity is reserved on thick edges only: Dashed thick edges correspond to Steiner trees, while full ones correspond to shortest paths.

(2) For each sender $s \in \mathcal{S}$, compute a $\rho_{\text {st }}$-approximate Steiner tree $T(s)$ on $\{s\} \cup$ $\mathcal{R}^{\prime}$, and add one unit of capacity to each edge of $T(s)$.

(3) Add one unit of capacity along the shortest path between each receiver $r \in \mathcal{R}$ and $\mathcal{R}^{\prime}$.

It remains to specify the path between each sender-receiver pair $(s, r)$. Assume that the shortest paths are computed with a consistent tie-breaking rule. Let $r^{*}$ be the receiver in $\mathcal{R}^{\prime}$ which is closest to $r$. The path $P_{s r}$ between $s$ and $r$ is obtained by concatenating the (simple) path between $s$ and $r^{*}$ in $T(s)$, with the shortest path between $r^{*}$ and $r$.

The thereby produced solution is not a tree solution. Though an optimal tree solution is a constant-factor approximation to an optimal graph solution, it is known [11] that an optimal solution to $($ AsymT $)$ is not an optimal solution to VPND. All previous constant-factor approximation algorithms for VPND, however [12, 7], produce tree reservations.

Before we proceed with the analysis of Algorithm 2, we state a corollary of Lemma 2. Here, given a subset $V^{\prime}$ of nodes, we denote the cost of the optimum Steiner tree on $V^{\prime}$ by $s t\left(V^{\prime}\right)$.

CoROllary 2 (see [12]). Let $\mathcal{R}_{1}, \mathcal{R}_{2}, \ldots, \mathcal{R}_{s}$ be a partition of $\mathcal{R}$ into $S$ (disjoint) subsets. Consider an arbitrary perfect matching between $\mathcal{S}$ and this family of subsets. Let $\mathcal{R}(s)$ be the subset matched with sender s. The sum over $\mathcal{S}$ of the costs of the optimum Steiner trees on $\{s\} \cup \mathcal{R}(s)$ is a lower bound on OPT:

$$
\sum_{s \in \mathcal{S}} s t(\{s\} \cup \mathcal{R}(s)) \leq O P T .
$$

Proof. This follows from Lemma 2 since a solution of a subinstance $\{s\}, \mathcal{R}(s)$ contains a Steiner tree with terminals $\{s\} \cup \mathcal{R}(s)$.

TheOREM 4. Algorithm 2 is a $\left(2+\rho_{\text {st }}\right) /\left(1-e^{-R / S}\right)$-approximation algorithm for VPND.

Theorem 4 is a straightforward consequence of the following lemmas.

LEMma 4. For a uniformly chosen random sender $s^{\prime}$,

$$
E\left[s t\left(\left\{s^{\prime}\right\} \cup \mathcal{R}^{\prime}\right)\right] \leq \frac{O P T}{S\left(1-e^{-R / S}\right)} .
$$

Proof. Consider the following random process. For each receiver $r$, we assign $r$ to a sender $s$ chosen uniformly at random. Let $\mathcal{R}(s)$ be the subset of receivers assigned to $s$. Note that the subsets $\mathcal{R}(s)$ partition $\mathcal{R}$ into $S$ (possibly empty) subsets. Thus, 
by Corollary 2 ,

$$
\sum_{s \in \mathcal{S}} s t(\{s\} \cup \mathcal{R}(s)) \leq O P T
$$

This means that, for the random sender $s^{\prime}$,

$$
E\left[s t\left(\left\{s^{\prime}\right\} \cup \mathcal{R}\left(s^{\prime}\right)\right)\right] \leq O P T / S .
$$

Let $A$ denote the event that $\mathcal{R}\left(s^{\prime}\right)$ is empty. By elementary probability theory,

$$
E\left[s t\left(\left\{s^{\prime}\right\} \cup \mathcal{R}\left(s^{\prime}\right)\right)\right]=P(A) E\left[s t\left(\left\{s^{\prime}\right\} \cup \mathcal{R}\left(s^{\prime}\right)\right) \mid A\right]+P(\bar{A}) E\left[s t\left(\left\{s^{\prime}\right\} \cup \mathcal{R}\left(s^{\prime}\right)\right) \mid \bar{A}\right] .
$$

Now observe that

$$
P(\bar{A})=1-(1-1 / S)^{R} \geq 1-e^{-R / S} .
$$

Moreover

$$
E\left[\operatorname{st}\left(\left\{s^{\prime}\right\} \cup \mathcal{R}\left(s^{\prime}\right)\right) \mid A\right]=E\left[\operatorname{st}\left(\left\{s^{\prime}\right\}\right)\right]=0 .
$$

Thus

$$
E\left[s t\left(\left\{s^{\prime}\right\} \cup \mathcal{R}\left(s^{\prime}\right)\right) \mid \bar{A}\right]=\frac{E\left[s t\left(\left\{s^{\prime}\right\} \cup \mathcal{R}\left(s^{\prime}\right)\right)\right]}{P(\bar{A})} \leq \frac{O P T}{S\left(1-e^{-R / S}\right)} .
$$

The claim follows by observing that, given $\bar{A}, \mathcal{R}\left(s^{\prime}\right)$ and $\mathcal{R}^{\prime}$ are identically distributed. Thus

$$
E\left[\operatorname{st}\left(\left\{s^{\prime}\right\} \cup \mathcal{R}^{\prime}\right)\right]=E\left[s t\left(\left\{s^{\prime}\right\} \cup \mathcal{R}\left(s^{\prime}\right)\right) \mid \bar{A}\right] \leq \frac{O P T}{S\left(1-e^{-R / S}\right)} .
$$

Lemma 5. The expected cost of the capacity installed in the second step of Algorithm 2 is at most

$$
\rho_{s t} O P T /\left(1-e^{-R / S}\right) .
$$

Proof. The expected cost considered is

$$
E\left[\sum_{s \in \mathcal{S}} c(T(s))\right],
$$

where $c(T(s))$ is the cost of the Steiner tree $T(s)$. Of course

$$
c(T(s)) \leq \rho_{s t} s t\left(\{s\} \cup \mathcal{R}^{\prime}\right) .
$$

Let $s^{\prime}$ be a sender chosen uniformly at random. By Lemma 4 ,

$$
\begin{aligned}
E\left[\sum_{s \in \mathcal{S}} c(T(s))\right] & \leq \rho_{s t} E\left[\sum_{s \in \mathcal{S}} s t\left(\{s\} \cup \mathcal{R}^{\prime}\right)\right]=\rho_{s t} S E\left[s t\left(\left\{s^{\prime}\right\} \cup \mathcal{R}^{\prime}\right)\right] \\
& \leq \rho_{s t} S \frac{O P T}{S\left(1-e^{-R / S}\right)}=\frac{\rho_{s t} O P T}{1-e^{-R / S}} .
\end{aligned}
$$


Lemma 6. The expected cost of the capacity installed in the third step of Algorithm 2 is at most

$$
2 O P T /\left(1-e^{-R / S}\right)
$$

Proof. Let $r^{\prime}$ be an arbitrary receiver in $\mathcal{R}^{\prime}$. Observe that the probability of any other receiver $r^{\prime \prime}$ being in $\mathcal{R}^{\prime}$, given that $r^{\prime}$ is in $\mathcal{R}^{\prime}$, is $1 / S$. In fact, let $\mathcal{R}^{*}$ be a random (possibly empty) partition element. Then

$$
\begin{aligned}
P\left(r^{\prime} \in \mathcal{R}^{\prime}\right) & =P\left(r^{\prime} \in \mathcal{R}^{*} \mid \mathcal{R}^{*} \neq \emptyset\right)=\frac{P\left(r^{\prime} \in \mathcal{R}^{*} \cap \mathcal{R}^{*} \neq \emptyset\right)}{P\left(\mathcal{R}^{*} \neq \emptyset\right)} \\
& =\frac{P\left(r^{\prime} \in \mathcal{R}^{*}\right)}{P\left(\mathcal{R}^{*} \neq \emptyset\right)}=\frac{1 / S}{1-(1-1 / S)^{R}} .
\end{aligned}
$$

By basically the same argument

$$
P\left(r^{\prime} \in \mathcal{R}^{\prime} \cap r^{\prime \prime} \in \mathcal{R}^{\prime}\right)=\frac{(1 / S)^{2}}{1-(1-1 / S)^{R}} .
$$

Thus

$$
P\left(r^{\prime \prime} \in \mathcal{R}^{\prime} \mid r^{\prime} \in \mathcal{R}^{\prime}\right)=\frac{P\left(r^{\prime \prime} \in \mathcal{R}^{\prime} \cap r^{\prime} \in \mathcal{R}^{\prime}\right)}{P\left(r^{\prime} \in \mathcal{R}^{\prime}\right)}=\frac{(1 / S)^{2} /\left(1-(1-1 / S)^{R}\right)}{(1 / S) /\left(1-(1-1 / S)^{R}\right)}=\frac{1}{S} .
$$

Now consider the following random process. In step $t$, let $\mathcal{A}_{t}$ be the subset of receivers considered so far, and let $\mathcal{B}_{t}$ be the bought receivers in $\mathcal{A}_{t}$. Initially $\mathcal{A}_{1}=\mathcal{B}_{1}=\left\{r^{\prime}\right\}$, where $r^{\prime}$ is a random receiver. In step $t$, we consider the receiver $r_{t} \in \mathcal{R} \backslash \mathcal{A}_{t}$ which is closest to $\mathcal{B}_{t}$, and we set $\mathcal{A}_{t+1}=\mathcal{A}_{t} \cup\left\{r_{t}\right\}$. Then, with probability $1 / S$, we buy $r_{t}$; that is,

- we add $S$ units of capacity along the shortest path from $r_{t}$ to $\mathcal{B}_{t}$, and

- we set $\mathcal{B}_{t+1}=\mathcal{B}_{t} \cup\left\{r_{t}\right\}$.

Otherwise, we rent $r_{t}$; that is,

- we add one unit of capacity along the shortest path from $r_{t}$ to $\mathcal{B}_{t}$, and

- we set $\mathcal{B}_{t+1}=\mathcal{B}_{t}$.

Note that, at the end of the process, the set of bought receivers $\mathcal{B}^{\prime}$ has the same distribution as the set $\mathcal{R}^{\prime}$ of selected receivers. Let $\ell\left(v, V^{\prime}\right)=\min _{v^{\prime} \in V^{\prime}}\left\{\ell\left(v, v^{\prime}\right)\right\}$ denote the minimum distance between a node $v$ and a subset of nodes $V^{\prime}$. The expected cost of the third step of the algorithm is upper bounded by the expected cost $c_{\text {rent }}$ of renting receivers

$$
\begin{aligned}
E\left[\sum_{r \in \mathcal{R} \backslash \mathcal{R}^{\prime}} \ell\left(r, \mathcal{R}^{\prime}\right)\right] & =E\left[\sum_{r_{t} \in \mathcal{R} \backslash \mathcal{B}^{\prime}} \ell\left(r_{t}, \mathcal{B}^{\prime}\right)\right] \leq E\left[\sum_{r_{t} \in \mathcal{R} \backslash \mathcal{B}^{\prime}} \ell\left(r_{t}, \mathcal{B}_{t}\right)\right] \\
& =E\left[\sum_{r_{t} \in \mathcal{R}}\left(1-\frac{1}{S}\right) \ell\left(r_{t}, \mathcal{B}_{t}\right)\right]=c_{\text {rent }},
\end{aligned}
$$

where the inequality comes from the fact that $\mathcal{B}_{t} \subseteq \mathcal{B}^{\prime}$ for any $t$. 
The expected cost $c_{b u y}$ of buying receivers is an upper bound on $c_{\text {rent }}$ :

$$
\begin{aligned}
c_{\text {rent }} & =E\left[\sum_{r_{t} \in \mathcal{R}}\left(1-\frac{1}{S}\right) \ell\left(r_{t}, \mathcal{B}_{t}\right)\right] \leq E\left[\sum_{r_{t} \in \mathcal{R}}\left(\frac{1}{S}\right) S \ell\left(r_{t}, \mathcal{B}_{t}\right)\right] \\
& =E\left[\sum_{r_{t} \in \mathcal{B}^{\prime}} S \ell\left(r_{t}, \mathcal{B}_{t}\right)\right]=c_{\text {buy }} .
\end{aligned}
$$

Note that $c_{b u y}$ is equal to $S$ times the expected cost of the Steiner tree on $\mathcal{B}^{\prime}$ which is obtained via the minimum spanning tree heuristic (starting Prim's algorithm from node $r^{\prime}$ ). It is well known that this heuristic provides a 2-approximate solution [17]. Thus

$$
c_{\text {buy }} \leq 2 S E\left[\operatorname{st}\left(\mathcal{B}^{\prime}\right)\right]=2 S E\left[\operatorname{st}\left(\mathcal{R}^{\prime}\right)\right]
$$

For a random sender $s^{\prime}$, by Lemma 4 ,

$$
E\left[s t\left(\mathcal{R}^{\prime}\right)\right] \leq E\left[s t\left(\left\{s^{\prime}\right\} \cup \mathcal{R}^{\prime}\right)\right] \leq \frac{O P T}{S\left(1-e^{-R / S}\right)} .
$$

Altogether,

$$
E\left[\sum_{r \in \mathcal{R}} \ell\left(r, \mathcal{R}^{\prime}\right)\right] \leq c_{r e n t} \leq c_{b u y} \leq 2 S \frac{O P T}{S\left(1-e^{-R / S}\right)}=\frac{2 O P T}{1-e^{-R / S}} .
$$

In section 3 we described a $(1+R / S)$-approximation algorithm. The factors $1+R / S$ and $\left(2+\rho_{s t}\right) /\left(1-e^{-R / S}\right)$ are equal for $R / S=2.78 \ldots<2.79$. Note that $1+R / S$ is increasing in $R / S$ and $\left(2+\rho_{s t}\right) /\left(1-e^{-R / S}\right)$ is decreasing in $R / S$. It follows that a combination (taking the minimum cost solution) of Algorithms 1 and 2 has an expected approximation guarantee of 3.79 , which is a considerable improvement compared to the 5.55-approximation ratio achieved by Gupta, Kumar, and Roughgarden $[12]$.

THEOREM 5. The combination (taking the cheaper solution) of Algorithms 1 and 2 is an expected 3.79-approximation algorithm for VPND.

5. Computing optimum Steiner trees. The performance ratios of Algorithms 1 and 2 meet roughly at $R / S \simeq 2.78$. In this case, the sampled set $\mathcal{R}^{\prime}$ of receivers has expected constant size. An optimum Steiner tree on a graph with $n$ nodes and $t$ terminals can be computed in $O\left(3^{t} n+2^{t} n^{2}+n^{3}\right)$ time with the DreyfusWagner algorithm [5]. This suggests the following variant of Algorithm 2, which computes optimal Steiner trees in step (2), instead of $\rho_{s t}$-approximate ones, whenever $\left|\mathcal{R}^{\prime}\right| \leq \log n$. Here $n$ is the number of nodes in the original graph $G$. Without loss of generality, we assume $n$ is sufficiently large.

Algorithm 3.

(1) Partition $\mathcal{R}$ into $S$ subsets uniformly at random. Select one subset $\mathcal{R}^{\prime}$ uniformly at random, among the nonempty subsets in the partition.

(2) For each sender $s \in \mathcal{S}$, compute a $\rho_{\text {st }}$-approximate Steiner tree $T(s)$ on $\{s\} \cup$ $\mathcal{R}^{\prime}$ if $\left|\mathcal{R}^{\prime}\right|>\log n$. Otherwise, compute an optimum Steiner tree $T(s)$ on $\{s\} \cup \mathcal{R}^{\prime}$. In both cases, add one unit of capacity to each edge of $T(s)$.

(3) Add one unit of capacity along the shortest path between each receiver $r \in \mathcal{R}$ and $\mathcal{R}^{\prime}$. 
Clearly, Algorithm 3 is a polynomial-time algorithm whose expected approximation guarantee is not worse than the one of Algorithm 2. In particular, if $R / S$ is very large, say, $R / S \geq \log \log n$, the approximation achieved is

$$
\frac{2+\rho_{s t}}{1-e^{-R / S}} \leq \frac{2+\rho_{s t}}{1-1 / \log n}=2+\rho_{s t}+o(1)<3.55 .
$$

What can be said about the approximation guarantee if $R / S \leq \log \log n$ ? In that case, the expected size of $\mathcal{R}^{\prime}$ is $1+(R-1) / S<1+\log \log n$. The probability that the size of $\mathcal{R}^{\prime}$ exceeds $\log n$ is at most $(1+\log \log n) / \log n$ by Markov's inequality. Thus with high probability Algorithm 3 computes optimum Steiner trees. When this happens, the approximation ratio is bounded by $3.325 \ldots<3.326$. In the very unlikely event that Algorithm 3 computes $\rho_{s t}$-approximate Steiner trees, the approximation guaranteed by Algorithm 1 alone is $O(\log \log n)$. Hence this event contributes to the expected approximation ratio with $o(1)$ only. This is the intuition behind the following theorem.

THEOREM 6. For a sufficiently large n, the combination (taking the cheaper solution) of Algorithms 1 and 3 is an expected 3.55-approximation algorithm for VPND.

Proof. Following the discussion above, if $R / S>\log \log n$, the expected approximation ratio achieved is $2+\rho_{s t}+o(1)<3.55$. Then we can restrict our analysis to the case $R / S \leq \log \log n$. Let $A P X$ denote the expected cost of the solution computed. One has

$$
A P X \leq E\left[\min \left\{(1+R / S) O P T, \sum_{s \in \mathcal{S}} c(T(s))+\sum_{r \in \mathcal{R}} \ell\left(r, \mathcal{R}^{\prime}\right)\right\}\right] .
$$

Of course,

$$
A P X \leq(1+R / S) O P T,
$$

and

$$
A P X \leq E\left[\sum_{r \in \mathcal{R}} \ell\left(r, \mathcal{R}^{\prime}\right)\right]+E\left[\min \left\{(1+R / S) O P T, \sum_{s \in \mathcal{S}} c(T(s))\right\}\right] .
$$

By Lemma 6,

$$
E\left[\sum_{r \in \mathcal{R}} \ell\left(r, \mathcal{R}^{\prime}\right)\right] \leq \frac{2 O P T}{1-e^{-R / S}}
$$

Let $A$ denote the event that $R^{\prime}=\left|\mathcal{R}^{\prime}\right| \leq \log n$. By elementary probability theory one has

$$
\begin{aligned}
E\left[\min \left\{(1+R / S) O P T, \sum_{s \in \mathcal{S}} c(T(s))\right\}\right] \leq & P(\bar{A}) E[(1+R / S) O P T \mid \bar{A}] \\
& +P(A) E\left[\sum_{s \in \mathcal{S}} c(T(s)) \mid A\right] .
\end{aligned}
$$


We now consider both terms separately. By Markov's inequality one has $P(\bar{A}) \leq$ $(1+\log \log n) / \log n$. Thus

$$
\begin{aligned}
P(\bar{A}) E[(1+R / S) O P T \mid \bar{A}] & \leq P(\bar{A}) E[(1+\log \log n) O P T \mid \bar{A}] \\
& \leq \frac{(1+\log \log n)^{2}}{\log n} O P T .
\end{aligned}
$$

Given $A$, Algorithm 3 computes optimal Steiner trees $T(s)$ of cost $s t\left(\{s\} \cup \mathcal{R}^{\prime}\right)$. Also $E\left[s t\left(\{s\} \cup \mathcal{R}^{\prime}\right) \mid R^{\prime} \leq h\right]$ is a nondecreasing function of $h$. Thus, from the proof of Lemma 5 , the second term on the right of (9) can be bounded by

$$
P(A) E\left[\sum_{s \in \mathcal{S}} c(T(s)) \mid A\right] \leq E\left[\sum_{s \in \mathcal{S}} s t\left(\{s\} \cup \mathcal{R}^{\prime}\right)\right] \leq \frac{O P T}{1-e^{-R / S}} .
$$

One therefore has

$$
\begin{aligned}
E\left[\min \left\{(1+R / S) O P T, \sum_{s \in \mathcal{S}} c(T(s))\right\}\right] & \leq \frac{(1+\log \log n)^{2}}{\log n} O P T+\frac{O P T}{1-e^{-R / S}} \\
& \leq\left(1+\frac{(1+\log \log n)^{2}}{\log n}\right) \frac{O P T}{1-e^{-R / S}} .
\end{aligned}
$$

Combining (7), (8), and (10), we conclude that

$$
A P X \leq \min \left\{(1+R / S) O P T,\left(3+\frac{(1+\log \log n)^{2}}{\log n}\right) \frac{O P T}{1-e^{-R / S}}\right\} .
$$

Thus, for a sufficiently large $n$, the expected approximation ratio for $R / S \leq \log \log n$ is upper bounded by $3.325 \ldots<3.326$. Altogether, the approximation ratio is

$$
\min \left\{3.326,2+\rho_{s t}+o(1)\right\}<3.55 .
$$

COROLLARY 3. There is an expected 3.55-approximation algorithm for VPND.

Proof. When $n$ is upper bounded by a constant, the optimum solution can be computed in polynomial time by trivial enumeration. The claim follows from Theorem 6.

\section{REFERENCES}

[1] M. Andrews And L. Zhang, The access network design problem, in Proceedings of the IEEE Symposium on Foundations of Computer Science, IEEE Computer Society, Los Alamitos, CA, 1998, pp. 40-49.

[2] B. Awerbuch and Y. Azar, Buy-at-bulk network design, in Proceedings of the IEEE Symposium on Foundations of Computer Science, IEEE Computer Society, Los Alamitos, CA, 1997, pp. 542-547.

[3] G. Brightwell, G. Oriolo, and F. B. Shepherd, Reserving resilient capacity in a network, SIAM J. Discrete Math., 14 (2001), pp. 524-539.

[4] G. Brightwell, G. Oriolo, And F. B. Shepherd, Reserving resilient capacity for a single commodity with upper-bound constraints, Networks, 41 (2003), pp. 87-96.

[5] S. E. Dreyfus And R. A. Wagner, The Steiner problem in graphs, Networks, 1 (1971/1972), pp. 195-207.

[6] N. G. Duffield, P. Goyal, A. Greenberg, P. Mishra, K. K. Ramakrishnan, and J. E. VAN DER MERWE, A flexible model for resource management in virtual private networks, in Proceedings of the ACH Conference on Applications, Technologies, Architectures, and Protocols for Computer Communication, Cambridge, MA, 1999, pp. 95-108. 
[7] F. Eisenbrand And F. GRandoni, An improved approximation algorithm for virtual private network design, in Proceedings of the ACM-SIAM Symposium on Discrete Algorithms, ACM, New York, SIAM, Philadelphia, 2005, pp. 928-932.

[8] F. Eisenbrand, F. Grandoni, G. Oriolo, and M. Skutella, New approaches for virtual private network design, in Proceedings of the International Colloquium on Automata, Languages and Programming, Lisbon, Portugal, 2005, pp. 1152-1162.

[9] S. Even, A. Itai, And A. Shamir, On the complexity of timetable and multicommodity flow problems, SIAM J. Comput., 5 (1976), pp. 691-703.

[10] J. A. Fingerhut, S. Suri, And J. S. Turner, Designing least-cost nonblocking broadband networks, J. Algorithms, 24 (1997), pp. 287-309.

[11] A. Gupta, J. Kleinberg, A. Kumar, R. Rastogi, and B. Yener, Provisioning a virtual private network: A network design problem for multicommodity flow, in Proceedings of the ACM Symposium on Theory of Computing, ACM, New York, 2001, pp. 389-398.

[12] A. Gupta, A. Kumar, And T. Roughgarden, Simpler and better approximation algorithms for network design, in Proceedings of the ACM Symposium on Theory of Computing, ACM, New York, 2003, pp. 365-372.

[13] T. C. Hu, Multi-commodity network flows, Oper. Res., 11 (1963), pp. 344-360.

[14] C. Hurkens, J. Keijsper, and L. Stougie, Virtual private network design: A proof of the tree routing conjecture on ring networks, in Proceedings of the International Conference on Integer Programming and Combinatorial Optimization, Berlin, Germany, 2005, pp. 407421.

[15] G. Italiano, S. Leonardi, and G. Oriolo, Design of networks in the hose model, in Proceedings of ARACNE, Rome, Italy, 2002, pp. 65-76.

[16] G. F. Italiano, R. RAstogi, and B. Yener, Restoration algorithms for virtual private networks in the hose model, in Proceedings of the Annual Joint Conference of the IEEE Computer and Communications Society, New York, NY, 2002, pp. 131-139.

[17] L. Kou, G. Markowsky, and L. Berman, A fast algorithm for Steiner trees, Acta Inform., 15 (1981), pp. 141-145.

[18] G. Robins AND A. Zelikovsky, Improved Steiner tree approximation in graphs, in Proceedings of the ACM-SIAM Symposium on Discrete Algorithms, ACM, New York, SIAM, Philadelphia, 2000, pp. 770-779.

[19] A. SchriJver, Combinatorial Optimization: Polyhedra and Efficiency, Algorithms and Combinatorics 24, Springer-Verlag, Berlin, 2003. 